\title{
Una Reflexión Metacognitiva de los Tipos de Pensamientos a Través de una Investigación Matemática. Convergencia Vs Divergencia
}

\section{Metacognitive Reflection of Thoughts Types across of Mathematical Research. Convergence Vs Divergence}

\author{
PhD. Mauro Misael García Pupo, \\ En Ciencias Matemáticas Universidad la Habana, Cuba, Director de Programas de maestría y doctorado en Educación Matemática \\ Universidad Antonio Nariño, Bogotá, Colombia. \\ mauro@uan.edu.co
}

Recebido: Junio 7 de 2013

Aceptado: Sept. 27 de 2013

\begin{abstract}
RESUMEN
Se hace una reflexión, sobre la riqueza del pensamiento matemático en la construcción de significados relevantes en una investigación matemática; a través de una reconstrucción metacognitiva de un problema de investigación concreto, después de más de veinte años. Para estos resultados se describen situaciones cognitivas, de diferentes tipos, que permiten recrear lo planteado por Henry Poncaré y su discípulo Hadamard quien explica ese ¿Cómo? Mediante una ampliación de lo conceptualizado por su maestro:Documentación (informarse, leer previamente, escuchar, discutir); Preparación (realizar un proceso de ensayo-error sobre diferentes vías e hipótesis, considerando un cambio eventual de actividad en caso de no obtener ningún progreso); Incubación (cambiar de actividad); Iluminación (ocurre la idea repentina); Verificación (la idea debe someterse al análisis y comprobación, al juicio crítico); Conclusión (ordenación y formulación rigurosa de los resultados.Precisamente el proceso en la etapa tres, permitió de forma repentina que aflorara "una idea" y así poder construir un significado abandonado semanas atrás. La trascendencia del mismo permitió una contribución sólida a la Teoría del Diseño de las Bases de Datos Relacionales y Deductiva; temática inmersa en la Matemática Computacional. "La formulación de un problema es frecuentemente más esencial que su solución, que puede ser tan solo un asunto de destreza matemática o experimental. Plantearse nuevas cuestiones, nuevas posibilidades, ver viejos problemas desde un nuevo ángulo, requiere una imaginación creadora y marca un avance real en la ciencia" Álvarez, E. (2010) citando a Albert Einstein (1938)
\end{abstract}

Palabras Clave: Metacognición, pensamiento divergente, pensamiento convergente, bases de datos.

\begin{abstract}
Becomes a reflection on richness the mathematical thinking in the construction of relevant meanings in a mathematical research; through a reconstruction of a research problem Metacognitive concrete, after more than twenty years. These results are described cognitive situations, different types, which allow you to recreate the issues raised by Henry Poncaré and his disciple, Hadamard, who explains that like? Through enlargement of the conceptualized by his master: documentation (please, read, listen, discuss); Preparation (be a process of trial-error on different routes and scenarios, considering a possible change of activity in case of not getting any progress); Incubation (change activity); Lighting (the sudden idea occurs); Verification (the idea must undergo analysis and verification, to critical judgement); Conclusion (management and rigorous formulation of the results. Precisely the process at stage three, allowed suddenly it launching "an idea" and to build a meaning abandoned weeks ago. The significance of the same allowed a solid contribution to the theory of the design of the relational database and deductive; immersed in the computational mathematical theme. "The formulation of a problem is often more essential than its solution, it may be just a matter of mathematical or experimental skill. "Raise new questions, new possibilities, to see old problems from a new angle, requires creative imagination and marks real progress in science" Alvarez, $e$. (2010) quoting Albert Einstein (1938).
\end{abstract}

Key Words: Metacognition, divergent thinking, convergent thinking. 


\section{INTRODUCCIÓN}

A finales de la década de los 60s, una reseña biográfica, de uno de los más grandes paradigmas del pensamiento científico del siglo pasado, inspiró con su traducción el inicio de este trabajo y decía: "...formular un enunciado verdadero de cualquier teoría exige mucha más creatividad que la demostración del mismo enunciado". Hoy, casi de medio siglo después, dado que me desarrollé en la carrera de Ciencias Matemáticas de la Universidad de Oriente en Cuba, hace que presente una reflexione acerca de ello , enfocado en el pensamiento matemático Se presenta en este artículo el pensamiento matemático desde una revisión de las perspectivas meta cognitivas de Poincaré y Hadamard de los llamados pensamiento convergente y pensamiento divergente y se contrastan aspectos de este complejo proceso con los resultados obtenidos como matemático en los en los cuales introduce otras apreciaciones alrededor del concepto de creatividad motivacional. García, M. (2006),

\section{APORTES DESARROLLOS}

En el primer resultado de la investigación "Dependencias Cartesianas en Bases de Datos" está el concepto y aporte teórico fundamental de la misma "Forma Normal Típica" FNT) (García, M. 1991). Lo allí conceptualizado sin ningún tipo de inconsistencia, requirió de varios intentos con aproximaciones totalmente incorrectas, que daban lugar a contraejemplos que impedían aplicaciones de los recursos teóricos que ya estaban construidos en el texto científico, hasta llegar a los resultados obtenidos, en la etapa final de la tesis.

Contaba con todo un cuerpo de definiciones, teoremas, y sus demostraciones ya consignadas en el documento científico en construcción, próximo a sustentar. Es decir, que todos los resultados teórico conceptuales de las buenas propiedades que las dependencias cartesianas $(D C)$ brindaban y que constituían una buena herramienta, para la realización de un buen diseño lógico de bases de datos relacionales (BDR) estaban ya demostrados. ( García, M. 1991)

Pero, el aporte central se quería centrarlo en las DC y sus propiedades para la introducción de una nueva Forma Normal muy cómoda y fácil de trabajar por los desarrolladores de BDR. Ya todos los miembros de los Seminarios de Bases de Datos e Inteligencia Artificial me habían sugerido que desistiera de continuar queriendo centrar el resultado en el concepto de FNT, que pusiera como aporte central el concepto de Dependencia Cartesiana y su aporte a una caracterización formalizada del concepto "entidad compartimentada" y las buenas propiedades que agregaba a las "dependencia multivaluadas" (DM) (Ullman, J.D.1982), por su oscura caracterización formal y la poca o casi imposibilidad de comprobación de su existencia en una BDR real. Además de reducir algoritmo de $\mathrm{O}\left(\mathrm{n}^{\mathrm{n}}\right)$ para una correcta descomposición de una relación a otro lineal, es decir, de $\mathrm{O}(\mathrm{n})$
Se estaba desarrollando un reto para una construcción de un significado que debía tener una verdadera contribución matemática a la Teoría del Diseño de las Bases de Datos Relacionales. La forma motivante que está expuesta por Ullman, J. (1982) se asumió como escuela y como metodología para el trabajo de investigación. Una investigación, de un matemático "puro" envuelto en las redes de las Ciencias de la Computación, requirió retomar más tarde el problema de inconsistencia de la definición de FNT, para apaciguar el desgaste sicológico. El tiempo propicio llegó en una madrugada y sentado en un sillón de un hospital como compañía de un familiar, que había sufrido un accidente, ya que se iluminó la solución buscada. Estaba el antecedente, en el momento de abandonar el enunciado inconsistente de FNT, formulado de la siguiente manera:

“DEFINICIÓN 4.1 (FORMA NORMAL TÍPICA): Sea R un esquema relacional y $D$ un conjunto de dependencias no triviales aplicable a $R$. Se dice que $R$ está en FNT si cuando exista una $D C X \rightarrow \rightarrow \rightarrow Y$, donde $Y$ no es vacío ni subconjunto de, $X$, y XY no incluye a todos los atributos de $R$, entonces $X$ es una súper llave para $R$." Son muchos los ejemplos (contraejemplos) que hace que esta definición falle y no permita constituirse en un aporte a la Teoría de las Formas Normales de las BDR. No viene al caso de que las explicite en este trabajo, pues no es el objetivo del mismo

Todo el problema radicaba que en la Teoría del diseño de las Bases de Datos Relacionales, con el descubrimiento de la existencia de las dependencias entre campos de cualquier relación $r$ para un esquema Relacional $\mathrm{R}$, surgieron desde el mismo momento que Codd, E.F. (1970) introduce el modelo relacional y fundamenta las Bases de Datos Relacionales (BDR).

Las primeras dependencias son las denominadas Dependencias Funcionales, luego las Dependencias Funcionales Parciales, las Dependencias Funcionales Transitivas y luego, las Dependencias Multivaluadas, introducidas por Fagin, R. (1977) quien aportaba a cada una de ellas una nueva forma normal y hacia lógico continuar en esta misma dirección, ejemplo de una metodología de investigaciones que venía desarrollándose desdela década de los 70s, elegante, sólida como soporte de la temática: Desde la primera forma normal hasta la cuarta, con una variante que incluye la tercera forma conocida por Forma Normal Boyce-Codd. (Ullman, J.D.1982).

El caso es que las DC eran DM con propiedades adicionales que permitiría poder enunciar las FNT en términos de la Cuarta Forma Normal soportada por la siguiente definición:

“DEFINICIÓN (CUARTA FORMANORMAL-4FN): Sea R un esquema relacional y $D$ un conjunto de dependencias no triviales aplicable $a R$. Se dice que R está en $4 F N$ si cuando exista una $D M X \rightarrow \rightarrow Y$, donde $Y$ no es vacio ni subconjunto de, $X, y X Y$ no incluye a todos los atributos de R, entonces X es una supe llave para R." (García, M. 1990).

Solo faltaba cerrar la definición de Cuarta Forma Normal en términos de DM, y el problema quedaba resuelto, tal como sigue: 
"DEFINICIÓN 4.1 (FORMA NORMAL TÍPICA): Sea R un esquema relacional y $D$ un conjunto de dependencias no triviales aplicable $a R$. Se dice que Restá en FNT si cuando exista una $D M X \rightarrow \rightarrow Y$, donde $Y$ no es vacio ni subconjunto de, $X, y X Y$ no incluye a todos los atributos de $R$, entonces $X$ es una supe llave para $R$; en caso contrario, es decir, $R=X Y$, entonces ó Xó Y son llaves simples para R." (García, M.(1991).

Con esto, estaba verificando las etapas tercera y cuarta formuladas por Hadamard a través de la experiencia:

I. Documentación (informarse, leer previamente, escuchar, discutir);

II. Preparación (realizar un proceso de ensayo-error sobre diferentes vías e hipótesis, considerando un cambio eventual de actividad en caso de no obtener ningún progreso);

III. Incubación, cambiar de actividad

IV. Iluminación, ocurre la idea repentina

V. Verificación, la idea debe someterse al análisis y comprobación, al juicio crítico

VI. Conclusión, ordenación y formulación rigurosa de los resultados

Es conveniente preguntarse: ¿Cuántos años transcurrieron para demostrar conjeturas o resolver algunos de los 23 problemas enunciados por David Hilbert en el Primer Congreso de Matemáticos en Paris? Por solo citar uno de los más recientes: la conjetura de Poincaré tardó muchos años en ser demostrada. Mucho más reciente, es la conjetura débil de Golbach. Por otra parte, el teorema en que demuestro que una DC es también una $\mathrm{DM}$, algo que era totalmente evidente, personalmente invertí bastante tiempo demostrarlo, quizás el mismo tiempo o más tiempo que el que tardé encontrar el concepto de FNT; con esta respuesta toma validez, de nuevo, la primera de las dos preguntas.

Entonces debíamos asegurar que: “demostrar es mucho más complejo que definir o enunciar". Por otra parte, el hecho de demostrar con esta definición de FNT formulada correctamente permitía cambiar un algoritmo cuya complejidad era del orden de $\mathrm{O}\left(\mathrm{n}^{\mathrm{n}}\right)$ y convertirlo en uno cuya complejidad era lineal $\mathrm{O}(\mathrm{n})$. Esta era una de las razones, más que suficiente para la decisión, más que justificada de esperar y olvidarme por un tiempo del problema que tenía que resolver. Con este ejemplo vuelve a tener una validez lo formulado por Einstein de forma sintética. Es decir, lleva a asegurar que: "definir o enunciar es mucho más complejo que demostrar".

Por tanto, ambas acciones cognitivas muestran un alto nivel de complejidad que le proporciona un carácter relativo a ambos argumentos en cuanto a su verdadero valor. Todo dependerá del nivel de complejidad de lo que se esté construyendo, de la persona que está realizando dichas acciones, del contexto académico, de factores psicoambientales, etc. Es decir, a veces, es más difícil resolver o demostrar que formular, enunciar o definir y viceversa. Creo que sobran los ejemplos. Además, Einstein en la traducción con la que se comienza este trabajo no es nada categórico cuando utiliza el adverbio "frecuentemente", en contraste con la interpretación totalmente cerrada que le di al comienzo de mi carrera, quizás no por mi culpa sino por la traducción de lo que leí en ese entonces. Todo gira alrededor de un problema y su solución. De eso se tratan las investigaciones matemáticas.

\section{¿Qué es la resolución de un problema?}

Miguel Cruz (2006) en su obra señala: "Desde una perspectiva personológica, la Resolución de Problemas constituye una actividad eminentemente consciente, y relativa a la cognición humana. Sin embargo, su análisis no puede estar completo si se margina el portador material del pensamiento. Por ello, antes de pasar al análisis gnoseológico, es conveniente abordar ciertos aspectos ontológicos del cerebro humano." (Cruz, M. 2006)

El cerebro humano. Más adelante continúa diciendo:..."Hoy día los neurocientíficos coinciden en que la naturaleza del pensamiento sigue siendo un enigma. No obstante, en el último siglo han aflorado disímiles resultados de relevante interés. Por ejemplo, en su Inside the Brain: Revolutionary Discoveries of How the Mind Works, el premio Pulitzer R. Kotulak llegó a la siguiente conclusión: "El cerebro no es un órgano estático; es una masa de conexiones celulares en constante cambio muy influida por la experiencia". En cualquier caso, la experiencia no es el único factor que incide en el cerebro; también influye en este órgano el pensamiento, pues los investigadores han comprobado que los individuos mentalmente activos tienen hasta un $40 \%$ más de conexiones (sinapsis) entre las neuronas que los mentalmente perezosos. Hay informes de que al envejecer se pierden algunas neuronas, y que en la tercera edad la memoria se debilita. De todos modos, la diferencia es mucho menor de lo que en un tiempo se pensaba. Un informe de National Geographic sobre el cerebro humano asevera: La gente mayor [...] conserva la capacidad de generar nuevas conexiones y mantener las antiguas mediante la actividad mental. Esto es impresionante, pues también se conoce que en toda una vida el hombre utiliza apenas una diezmilésima parte del potencial del cerebro."

El cerebro está compuesto por neuronas, cuyas interconexiones determinan la forma de cómo funciona el cerebro como centro regulador de cada persona. En la figura 2 se puede observar los diferentes tipos de estructura que pueden presentar éstas:

No se quie5re hacer un discurso sobre las diferentes dimensiones y definiciones que tiene el concepto "problemas" o "resolución de problemas". El punto de interés es detenerse a resolver que tipo de pensamiento es el que ocurre en un sujeto cuando está afrontando "un problema" y comienza a darle "solución".

Está claro en la literatura, desde cualquier perspectiva que se mire, de que se trata de un proceso muy complejo y que de hecho es un sistema complejo. 
Figura 1: Estructura y funciones del encéfalo humano

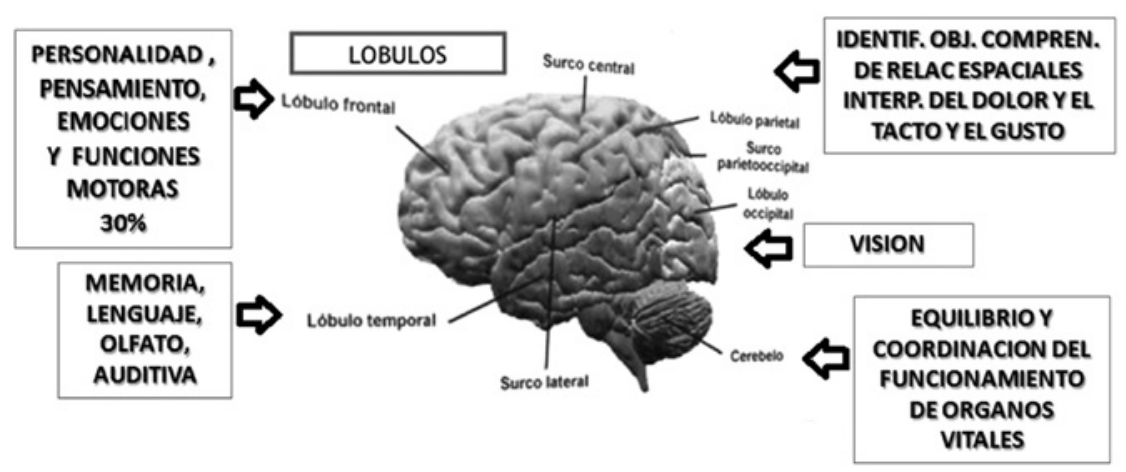

Fuente: Recuperado el 22 de febrero de 2013 del URL:http://www.slideshare.net/analobello/fisiologia-del-cerebro

Figura 2: Estructura de una neurona y sus relaciones

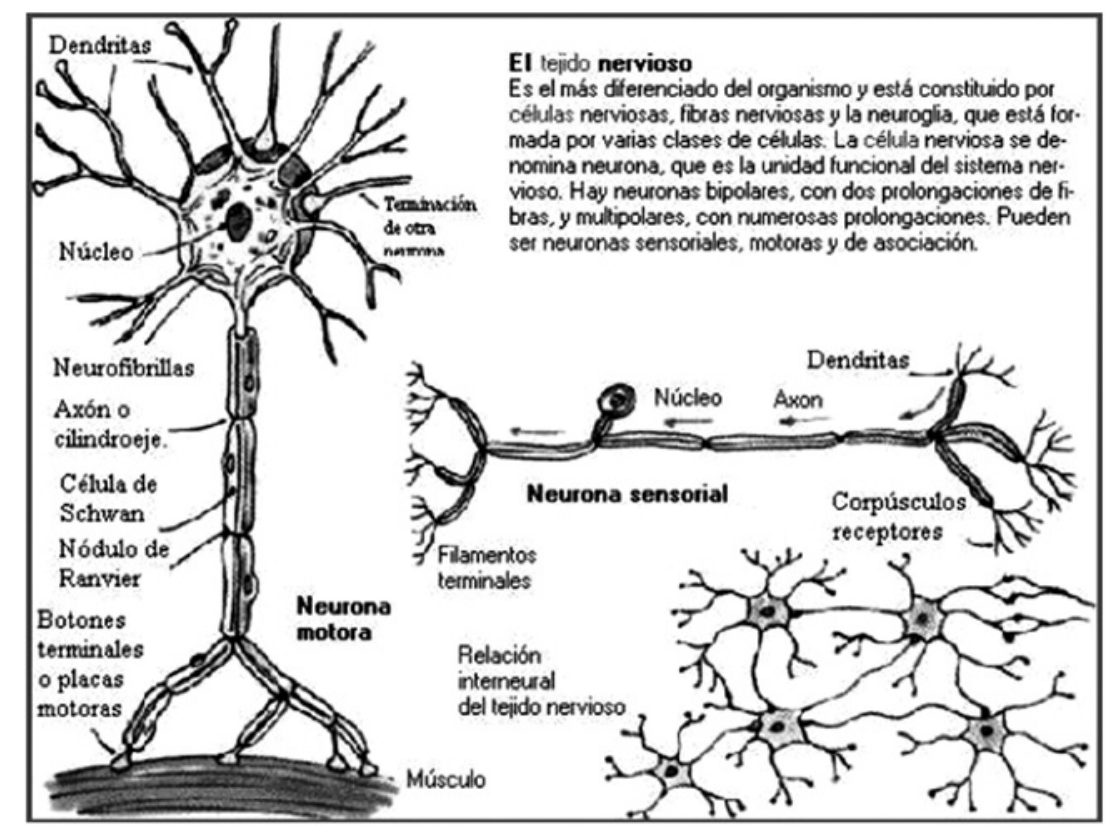

Fuente: Recuperado el 22 de febrero de 2013 en: https://www.google.com/search?q=estructura+ de+una+neurona+humana\&hl=e s\&site=webhp\&tbm=isch\&tbo=u\&source=univ\&sa =X\&ei=wHK CUeagJcfW0QG84IGoBw\&ved=0CDkQsAQ\&biw=1271\&bih=815

Se hacen apreciaciones sobre el concepto de creatividad en las matemáticas. Así como, no se tiene interés de caracterizar o de comentar las interrelaciones existentes entre el concepto de creatividad y su necesaria asociación con otros aspectos que caracterizan esencialmente al ser humano, tales como:

- "Creatividad e inteligencia

- Creatividad y personalidad

- Creatividad y cultura

- Creatividad y motivación"
Por otra parte, abordar la resolución de un problema matemático es casi imposible sin una clarificación del pensamiento convergente y divergente..."no es posible un abordaje del proceso de Resolución de Problemas al margen de la forma en que se despliega el pensamiento correcto. La mayoría de los autores delimitan dos tipos de pensamiento, los cuales sirven para caracterizar los esquemas de razonamiento que tienen lugar. Por una parte se distingue el pensamiento convergente, el cual se erige a través de operaciones lógicas claramente identificables. Es predominantemente deductivo y esperable a priori. Por otra parte se encuentra el pensamiento 
divergente, matizado por acciones predominantemente inductivas, creativas por naturaleza."1 ( Cruz, M 2006).

\section{CONCLUSIONES}

Sobre las características de los diferentes tipos de pensamientos que ocurren en la construcción matemática son muchos los autores que tratan de acercarse a los mismos desde disciplinas y perspectivas diferentes.

Se considera de enorme importancia un acercamiento a la caracterización del pensamiento matemático que ocurre en los procesos académicos, es por ellos que debe considerarse:

- La naturaleza de los diferentes temas que se abordan

- Las condiciones socio-psicológicas que existe en el contexto

- Las características de los estudiantes y del grupo en general.

- El éxito o fracaso que preceden al tema o problema en cuestión

Se establece que no siempre para problemas análogos ocurren una misma forma cognitiva del pensamiento, incluso para un mismo sujeto. Con los ítems anteriores se está tratando de suavizar este problema, pero está claro que su naturaleza es compleja y aunada a la individualidad o el carácter singular del pensamiento que caracteriza a cada ser humano y en que el estudiante del salón de una clase de matemática no es ajeno. Además, del carácter subjetivo y abstracto de esta disciplina.

Con la construcción de la definición de Forma Normal Típica se puede afirmar que el pensamiento matemático ocurrido, en ese momento dado, fue de carácter divergente, mientras que la búsqueda de la demostración de que las dependencias cartesianas constituían dependencias multivaluadas y en el resto de los resultados tuvo un carácter convergente

Miguel Cruz (2006). La enseñanza de las matemáticas a través de la resolución de problemas. Órgano editor Educación Cubana.

\section{Referencias}

Álvarez, E. (2010). Creatividad y Pensamiento Divergente Desafío de la mente o desafío del ambiente, InterAC. Recuperado el 28 de febrero de 2013 del URL: http://www.interac.es/adjuntos/ creapensa_diver.pdf

Codd, E.F. (1970). A Relational Model for Large Shared Data Bank, ACM Communication, Vol 13, no 6, 377-387

Cruz, M. (2006). La enseñanza de las matemáticas a través de la resolución de problemas. Órgano editor Educación Cubana. p.p. 39-42

Fagin, R. (1977). Multivalued Dependencies and New Normal Form for Relational Database. ACM Transaction on Database Systems, Vol 2, no 3,,262-278.

García, M. (1990). Dependencias cartesianas en bases de datos. Tesis en opción al grado científico de doctor en Ciencias Matemáticas (Ph.D.). Universidad de la Habana. pp. 29.

(1991). Las dependencias cartesianas y una nueva variante de forma normal para bases de datos relacionales. Revista Ciencias Matemáticas, Universidad de la Habana Vol. XII, Nos. 2,3.p. 154.

(2006). Filiations and ruptures with the redundancy on Mathematics and Computer Sciences. International Congress of Mathematicians, ICM 2006. Madrid, Agosto 22-30 de 2006.

Guilford, J.P.(1978). La creatividad: presente, pasado y futuro. Paidós. Buenos Aires

Hadamard, J (1947). Psicología de la invención en el campo matemático. Espasa-Calpe. Buenos Aires.

Poincare, H (1908). L’Enseignement Mathématique

Tall, D. y Gray, E. (1991). Duality, Ambiguity and Flexibility in Successful Mathematical Thinking. Proceedings of PME 15, Assisi, 2 72-79.

Tall D. (2012). Making Sense of Mathematical Reasoning and Proof. Plenary at Mathematics \& Mathematics Education: Searching for Common Ground: A Symposium in Honor of Ted Eisenberg, April 29-May 3, 2012, Ben-Gurion University of the Negev, Beer Sheva, Israel.

Ullman, J.D.(1982). Principles of Database Systems. Computer Science Press. Palo Alto, California. 